UN ACERCAMIENTO EMPÍRICO A LA CURVA DE PHILLIPS: HONDURAS

Revista Economía y Administración, Vol. 9, No.1, 2018

UN ACERCAMIENTO EMPÍRICO A LA CURVA DE PHILLIPS: HONDURAS

\author{
Adony Ordoñez Espinal \\ Universidad Nacional Autónoma de Honduras (UNAH) \\ Pedro Zuniga Figueroa \\ Universidad Nacional Autónoma de Honduras (UNAH) \\ Carrera de Economía \\ Ciudad Universitaria, edificio C1, segundo piso, Tel./ Cel. (504) 9765-6636 \\ pedroxzuniga@hotmail.com \\ (1) Víctor Ordoñez Portillo \\ Universidad Nacional Autónoma de Honduras (UNAH) \\ Carrera de Economía \\ Ciudad Universitaria, edificio C1, segundo piso, Tel./ Cel. (504) 9715-2036 \\ ordonezportillov85@hotmail.es
}

DOI: $\underline{\text { http://dx.doi.org/10.5377/eya.v9i1.6655 }}$

\title{
RESUMEN
}

El debate sobre la relación entre inflación y desempleo sigue vigente, en la presente investigación se aborda esta temática en primera instancia de manera teórica y consecuentemente de forma empírica considerando la determinación de una tasa natural de desempleo y una correlación estadística para el periodo de tiempo estudiado.

Palabras clave: Inflación, Desempleo, Curva de Phillips, Tasa Natural de Desempleo. 
UN ACERCAMIENTO EMPÍRICO A LA CURVA DE PHILLIPS: HONDURAS

Revista Economía y Administración, Vol. 9, No.1, 2018

\title{
AN EMPIRICAL APPROACH TO THE PHILLIPS CURVE: HONDURAS
}

\author{
Adony Ordoñez Espinal \\ Universidad Nacional Autónoma de Honduras (UNAH) \\ Pedro Zuniga Figueroa \\ Universidad Nacional Autónoma de Honduras (UNAH) \\ Carrera de Economía \\ Ciudad Universitaria, edificio C1, segundo piso, Tel./ Cel. (504) 9765-6636 \\ pedroxzuniga@hotmail.com \\ (1) Víctor Ordoñez Portillo \\ Universidad Nacional Autónoma de Honduras (UNAH) \\ Carrera de Economía \\ Ciudad Universitaria, edificio C1, segundo piso, Tel./ Cel. (504) 9715-2036 \\ ordonezportillov85@hotmail.es
}

DOI: http://dx.doi.org/10.5377/eya.v9i1.6655

\begin{abstract}
The debate about the relationship between

inflation and unemployment is still valid,

in the present investigation this subject is

addressed in a theoretical way and

consequently in an empirical way

considering the determination of a natural

unemployment rate and a statistical

correlation for the period studied.
\end{abstract}

Keywords: Inflation, Unemployment,

Phillips Curve, Natural Unemployment

Rate. 


\section{UN ACERCAMIENTO EMPÍRICO A LA CURVA DE PHILLIPS: HONDURAS}

Revista Economía y Administración, Vol. 9, No.1, 2018

\section{INTRODUCCIÓN}

El presente documento tiene como objetivo el estudio de la relación entre inflación y desempleo mediante la estimación de la curva de Phillips en Honduras, para este propósito es menester comenzar con una revisión histórica de los conceptos que forman parte del análisis de la curva de Phillips, del mismo modo es preciso exponer las diferentes interpretaciones que se le han dado al concepto junto con los cambios y críticas que ha recibido.

Para la determinación de la misma se hará uso de herramientas estadísticas y econométricas que permitirán comprobar la existencia de una relación negativa y causal entre inflación y desempleo, para dicha tarea se tomará como referencia el periodo 1990-2016 en Honduras; este análisis estadístico a la vez posibilitará una estimación de la Tasa Natural de Desempleo (TND) y la evaluación de la capacidad predictiva de la curva de Phillips, lo cual sin duda alguna puede ser de utilidad para la evaluación y elaboración de política económica.

\section{PLANTEAMIENTO DEL PROBLEMA}

Un nivel de precios estable es indispensable para mantener un crecimiento económico sostenible, del mismo modo para generar desarrollo económico es necesario promover un nivel de empleo que vaya acorde a los requerimientos de la sociedad en general; desde hace mucho tiempo se ha estudiado la relación que existe entre ambas variables, en este sentido, fue Phillips (1958) quien en primera instancia demostró con evidencia empírica la relación inversa entre los salarios nominales y el desempleo, este análisis ha ido evolucionando con el tiempo y con los problemas surgidos a lo largo de la segunda mitad del siglo XX e inicios del siglo XXI. Tal es el caso de la crítica de Friedman (1968; 1977) y Phelps (1968a; 1968b) a la curva de Phillips original ya que no incluía las expectativas, lo que dio lugar a una nueva curva de Phillips aumentada en expectativas, posteriormente se incluyeron más variables en la curva de Phillips neo-keynesiana, aspectos 


\section{UN ACERCAMIENTO EMPÍRICO A LA CURVA DE PHILLIPS: HONDURAS}

Revista Economía y Administración, Vol. 9, No.1, 2018

que serán analizados a detalle posteriormente.

Una característica muy importante de la curva de Phillips es que ha sido blanco de numerosas críticas por lo que al abordar este tema se debe tener en cuenta que es preciso analizar todas las perspectivas por las cuales se ha estudiado esta hipótesis.

Por otra parte, un aspecto importante entorno a la curva de Phillips es la hipótesis de la tasa natural de desempleo (TND), sobretodo su aproximación empírica que parte de la ecuación de la curva de Phillips aumentada con expectativas, aporte teórico elaborado por Friedman (1968), y que en estudios más actuales ha sido calculada a través del filtro Hodrick-Prescott.

Sin embargo, el análisis de estas variables no se ha realizado de forma conjunta en Honduras, problema que hasta cierto punto limita el campo de estudio no solo en el ámbito académico y científico, sino que también en la elaboración de política económica que promueva la estabilidad de precios y el equilibrio en el mercado laboral, cuestiones que a la larga se traducen en mayores tasas de crecimiento económico.

\section{JUSTIFICACIÓN}

El tema de la curva de Phillips ha sido muy poco estudiado en Honduras, a pesar de su relevancia teórica y empírica no se encuentran estudios publicados que traten de este tema en específico, si bien es cierto existen documentos que determinan una ecuación de nivel de precios en base a la curva de Phillips, el análisis de la misma no es el objetivo primordial de estos estudios. El poco conocimiento acerca de esta relación es una razón importante para emprender un estudio sobre este tema; a su vez, al tratar la curva de Phillips resulta necesario presentar de forma apropiada la discusión teórica a su alrededor y estudiar la factibilidad de los conceptos subsecuentes a ese "trade-off" entre inflacióndesempleo para comprobar si se pueden contrastar con la evidencia empírica de Honduras.

El contexto actual del mercado laboral es complejo, con una tasa de desempleo en aumento en los últimos 
UN ACERCAMIENTO EMPÍRICO A LA CURVA DE PHILLIPS: HONDURAS

Revista Economía y Administración, Vol. 9, No.1, 2018

años y un gran porcentaje de la población en condiciones de subocupación, resulta necesario analizar posibles alternativas para la toma de decisiones que favorezcan a una mejor asignación de los recursos que al final repercutan positivamente en la economía nacional, para ello un análisis de la Curva de Phillips y la estimación de la tasa natural de desempleo (TND) puede representar una herramienta importante y sentar un precedente para futuros estudios.

\section{PREGUNTAS}

DE

\section{INVESTIGACIÓN}

¿Existe una relación entre inflación y desempleo en Honduras durante el periodo 1990-2016?

¿Cuál es la tasa natura de desempleo (TND) para Honduras durante el periodo 1990-2016?

¿Sería eficaz tomar en cuenta la curva de Phillips como herramienta de política económica?

\section{OBJETIVO GENERAL}

Determinar la relación que existe entre inflación y desempleo, y las principales implicancias que esta pueda tener en Honduras durante el periodo 1990-2016.

\section{Objetivos Específicos}

Estimar la curva de Phillips para Honduras durante el periodo 19902016.

Estimar la tasa natural de desempleo en Honduras durante el periodo 1990-2016.

Analizar la eficacia de la curva de Phillips como herramienta de política económica en Honduras.

\section{Hipótesis de Investigación}

$\mathrm{H}_{0}$ : Existe una relación negativa entre inflación y desempleo a corto plazo en Honduras.

$\mathrm{H}_{1}$ : No existe una relación negativa entre inflación y desempleo a corto plazo en Honduras.

$\mathrm{H}_{0}$ : Es posible hacer una aproximación empírica al concepto de TND en Honduras.

$\mathrm{H}_{1}$ : No es posible hacer una aproximación empírica al concepto de TND en Honduras.

$\mathrm{H}_{0}$ : Es eficaz utilizar la curva de Phillips como una herramienta para la elaboración de política. 
UN ACERCAMIENTO EMPÍRICO A LA CURVA DE PHILLIPS: HONDURAS

Revista Economía y Administración, Vol. 9, No.1, 2018

$\mathrm{H}_{1}$ : No es eficaz utilizar la curva de

Phillips como una herramienta para la elaboración de política.

\section{TEORÍA/CÁLCULOS}

\section{Desarrollo de la Curva de Phillips}

El primer aporte remarcable fue realizado por Phillips (1958), quien a través de un estudio empírico relacionó la tasa de incremento de los salarios nominales con la tasa de desempleo, para esto tomó datos de Reino Unido del periodo 1861-1957, manifestando que la relación existente para estas variables presenta una forma no lineal (hiperbólica), es decir, que el vínculo entre estas variables es negativo o inverso mostrando una clara tendencia; cuando la tasa de salarios nominales es alta el desempleo es bajo, y es baja o negativa cuando el desempleo es alto.

Tiempo después Samuelson y Solow (1960), tomaron como referencia el planteamiento inicial de Phillips y lo redimensionaron proponiendo la siguiente pregunta: $¿ \mathrm{El}$ incremento salarial precede a la inflación o viceversa?, en este sentido, optaron por estudiar la relación entre inflación y desempleo utilizando datos de Estados Unidos; los resultados obtenidos expresaron una relación negativa entre estas variables y dieron más valor a la hipótesis inicial de Phillips, lo que permitió a los hacedores de política llevar a cabo acciones para estimular el empleo conociendo el efecto que puede tener esta medida en los precios; además fue gracias a estos autores que se dio nombre a lo que hoy conocemos como curva de Phillips.

En la Figura No. 1 se puede apreciar la relación inversa entre inflación y desempleo, donde mayores niveles de desempleo presionan a una baja en la inflación y viceversa, a su vez presenta una leve convexidad, tal y como lo manifestó Phillips en su exposición empírica sobre este "trade-off". 


\section{UN ACERCAMIENTO EMPÍRICO A LA CURVA DE PHILLIPS: HONDURAS}

Revista Economía y Administración, Vol. 9, No.1, 2018

Figura No. 1 Curva de Phillips

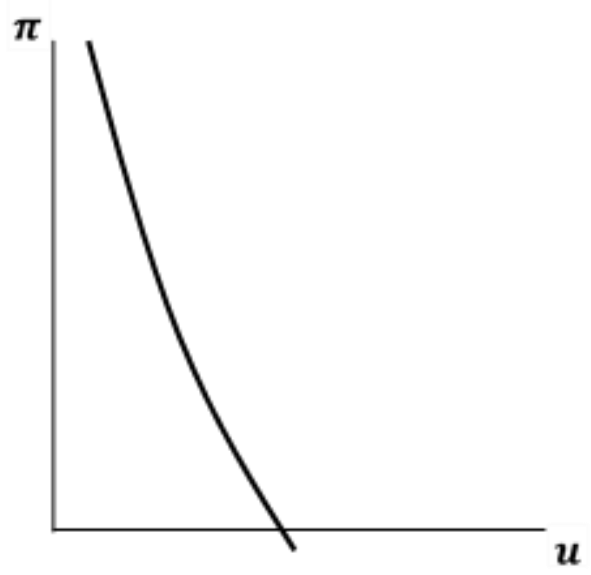

Fuente: Analytical Aspects of AntiInflation Policy, 1960 (p. 192), Samuelson y Solow.

Sin embargo, Phelps (1968a; 1968b) encontró ciertas debilidades en la afirmación de Solow y Samuelson, una de ellas es la inestabilidad de los precios, puesto que los agentes económicos anticipan el incremento en los precios, es decir, toman en consideración la inflación esperada cuando realizan sus decisiones, por lo cual se hizo necesario incluir al modelo original la inflación con expectativas adaptativas, esto significa que los individuos forman sus expectativas de inflación, a partir de la inflación pasada, y éstas se van corrigiendo en función de los errores cometidos; esta adhesión al bosquejo inicial se conoce con el nombre de curva de Phillips aumentada, conjuntamente propuso en su modelo el concepto de tasa de desempleo de estado estacionario que más adelante se conocería como tasa natural de desempleo.

Friedman (1968; 1977), coincidió con Phelps en que la curva de Phillips original carecía de algunos elementos importantes es por eso que critica la inestabilidad de la relación entre ambas variables (inflación y desempleo) en el largo plazo, en torno a este argumento formuló el concepto de tasa natural de desempleo (TND) la que definió como una tasa que es consistente con un determinado nivel de precios (equilibrio de ecuaciones walrasiano), esta TND es una línea vertical y el hecho que sea llamada "natural" no tiene nada que ver con que esta tasa sea inmutable o invariable puesto que muchas de las características de la estructura de mercado son determinadas y formuladas por la acción del hombre, de hecho, llamo a esta tasa "natural" para separar las fuerzas reales de las fuerza monetarias; otro aporte importante fue la diferenciación entre la curva de Phillips de corto plazo y largo plazo, donde en el corto plazo se podría 


\section{UN ACERCAMIENTO EMPÍRICO A LA CURVA DE PHILLIPS: HONDURAS}

Revista Economía y Administración, Vol. 9, No.1, 2018

generar este trade-off (inflacióndesempleo), mientras que en el largo plazo y asumiendo una TND este intercambio no tendría lugar y cualquier política encaminada a reducir el desempleo para estimular la demanda agregada solo generaría inflación. También observo que en algunos casos la relación entre inflación y desempleo podía ser positiva, este planteamiento fue producto del fenómeno de la estanflación ocurrido a principios de los años setenta cuando algunos países desarrollados sufrieron altas tasas de inflación aunadas a altos niveles de desempleo, esta relación positiva también puede ser producto de un cambio en el sistema monetario que rige a un país.

En la Figura No. 2 se rebate el planteamiento inicial de la curva de Phillips, demostrando que si bien es cierto a corto plazo se puede alterar el nivel de desempleo efectivo $(\overline{\boldsymbol{u}})$ por debajo de la tasa natural $\left(\boldsymbol{u}^{*}\right)$, a largo plazo solo implicaría un incremento en la inflación ya que la tasa de desempleo tendería a su equilibrio (TND), por ende todas las políticas encaminadas a mantener el desempleo por debajo de la TND solo provocarían un incremento en el nivel de precios, en detrimento del poder adquisitivo de los individuos, desestabilizando el ahorro y la inversión, por tanto, este escenario difiere (es peor) al inicial y puede provocar una espiral inflacionista; a este análisis también se le conoce como hipótesis aceleracioncita de la inflación y fue la base teórica utilizada para demeritar a la curva de Phillips como herramienta de política en los años 70's; sin embargo, el debate sigue vigente y no pierde relevancia en el análisis económico contemporáneo.

Figura No. 2 Curva de Phillips Aumentada

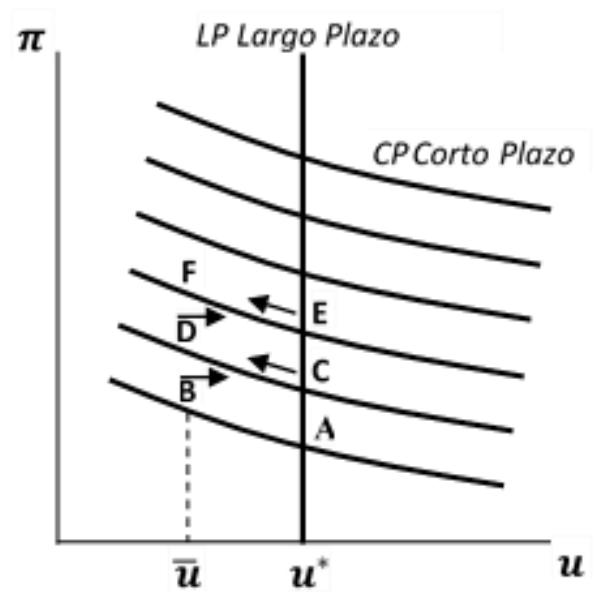

Fuente: Inflation and Unemployment, 1977 (p. 457), Friedman.

Alrededor de esta discusión sobre la tasa natural de desempleo se desarrollaron casi paralelamente 


\section{UN ACERCAMIENTO EMPÍRICO A LA CURVA DE PHILLIPS: HONDURAS}

Revista Economía y Administración, Vol. 9, No.1, 2018

otras teorías que ayudaron a explicar

el comportamiento a largo plazo de la relación de la curva de Phillips, alejándose un poco del paradigma neoclásico, por ejemplo, Modigliani y Papademos (1975) en su intento de explicar el fenómeno de la estanflación formularon un concepto alternativo al de la TND al que denominaron NIRU (noninflacionary rate of unemployment), este concepto es definido como una tasa tal que si el desempleo se encuentra por debajo de ella, conllevaría a una presión inflacionaria y viceversa, es decir, una tasa optima en la que la existencia de desempleo no altere de manera significativa la inflación en el largo plazo, entonces en el largo plazo la curva de Phillips seria representada por una línea vertical.

Luego Tobin $(1980 ; 1997)$ renombró la "non-inflacionary rate of unemployment" a NAIRU, dejando intacta su esencia, pero ampliando su base teórica; un rasgo importante es la diferenciación conceptual entre la tasa natural de desempleo y la NAIRU, en primer lugar, la TND parte de un equilibrio walrasiano donde los mercados están en equilibrio y el mercado de trabajo está siendo compensado por los precios y los salarios existentes llevando a la conclusión que la TND es una tasa de pleno empleo, en segundo lugar, la NAIRU es resultado de una economía con desequilibrios en los mercados, ya sea por excesos de demanda o excesos de oferta, entonces la NAIRU es una tasa de desempleo en la que el aumento de la inflación de los mercados de demanda excesiva equilibran los impactos que disminuyen la inflación de los mercados de oferta excesiva; por otra parte, la NAIRU puede presentar diferentes puntos de equilibrio, unos más eficientes que otros $\mathrm{y}$ no necesariamente óptimos.

El laureado nobel de economía Joseph Stiglitz (1997), reconoce la importancia y utilidad del uso de una NAIRU, como instrumento conceptual para entender las causas de la inflación, también como evidencia empírica para predecir posibles alteraciones en los precios, así como una herramienta de política económica; en el contexto en el cual 
UN ACERCAMIENTO EMPÍRICO A LA CURVA DE PHILLIPS: HONDURAS

Revista Economía y Administración, Vol. 9, No.1, 2018

se encontraba la economía estadounidense en los años 90’s con bajos niveles de inflación y bajo desempleo Stiglitz teorizo que la respuesta a dicho fenómeno se debía a una disminución en la tasa natural de desempleo (cabe recalcar que él considera la TND y la NAIRU como sinónimos); además postuló que las posibles causas de un cambio en la NAIRU provenían de aspectos estructurales como la productividad laboral y el cambio demográfico.

Años más tarde con el desarrollo de modelos de equilibrio general dinámico-estocástico se reformulo el planteamiento de la curva de Phillips ya que se le añadieron diferentes elementos que contemplan: la rigidez de precios, la utilización de parámetros microfundados (costo marginal y costo marginal esperado), precio del petróleo, y el cambio de expectativas adaptativas a expectativas racionales en contraposición a la ecuación presentada por Phelps y Friedman, es decir, las expectativas adaptativas consideran la inflación del pasado para las decisiones del presente mientras que las expectativas racionales consideran la inflación futura como elemento para la toma de decisiones del presente. (Clarida, Galí y Gertler, 1999; Galí y Gertler 1999; Roberts, 1995)

\section{METODOLOGÍA}

\section{Diseño de la Investigación}

La investigación tiene un diseño no experimental debido a que no intenta manipular la variable dependiente, sino estudiarla sin modificación alguna; y corte transversal con carácter retrospectivo para analizar la trayectoria y comportamiento de las variables durante el periodo 1990-2016.

\section{Alcance de la Investigación}

El trabajo de investigación posee un alcance explicativo, ya que pretende medir variables como la inflación y desempleo, para establecer un vínculo entre ambas, y así explicar la evolución, comportamiento e impacto.

\section{Recolección de Datos}

Los datos que utilizar provienen de fuentes secundarias, puesto que se obtuvieron de publicaciones de los 
UN ACERCAMIENTO EMPÍRICO A LA CURVA DE PHILLIPS: HONDURAS

Revista Economía y Administración, Vol. 9, No.1, 2018

indicadores económicos del Banco

Central de Honduras (inflación e inflación rezagada) y del Instituto

Nacional de Estadística (desempleo).

\section{Análisis de Datos}

La serie de datos existente da lugar a un análisis histórico sobre las variables incluidas en la investigación; este análisis se hará en base a la observación de los datos (serie anual de 27 observaciones) y a la elaboración de dos modelos econométricos que expliquen la relación y el comportamiento de las variables mediante la ecuación de la curva de Phillips aumentada donde únicamente se considera que la inflación está en función de la tasa de desempleo, la tasa natural de desempleo y la inflación esperada (expectativas adaptativas), con los respectivos parámetros estadísticos que permitirán posteriormente demostrar que tipo de relación tienen la inflación y el desempleo. Para conocer la capacidad predictiva del modelo se aplicarán los test de cointegración, raíz unitaria, normalidad, autocorrelación, heterocedasticidad y variables omitidas; por otra parte, para la determinación de la tasa de natural de desempleo se emplearán dos metodologías, una utilizada por Henao y Rojas (1998); Suarez y Cueva (2011) donde la TND se obtiene a partir de una ecuación de curva de Phillips aumentada y en segundo lugar una metodología utilizando el filtro Hodrick-Prescott (1997); y por último para determinar el tipo de relación que existe entre las variables se hará uso de la prueba de causalidad de Granger (1969).

\section{RESULTADOS}

Estimación de la Curva de Phillips, Tasa Natural de Desempleo y Prueba de Causalidad

Para la estimación de la curva de Phillips aumentada con expectativas se elaboraron dos modelos: la ecuación [Ec. (1)] o modelo lineal de curva de Phillips y la ecuación [Ec. (2)] o modelo semi-logarítmico de curva de Phillips; los modelos poseen distintas especificaciones del nivel de precios: la Ec. (1) tiene el valor del índice de precios al consumidor a diciembre de cada año, mientras que la Ec. (2) considera la 


\section{UN ACERCAMIENTO EMPÍRICO A LA CURVA DE PHILLIPS: HONDURAS}

Revista Economía y Administración, Vol. 9, No.1, 2018

tasa de variación interanual del índice de precios al consumidor. Las ecuaciones están representadas por:

Ec. (1)

$$
p=c-\beta_{0} u+\beta_{1} p_{t-1}+e_{i}
$$

Donde $\boldsymbol{p}$ son los precios de la economía medidos por el índice de precios al consumidor, $\boldsymbol{c}$ es una constante, $\begin{array}{llllll}\boldsymbol{\beta}_{\mathbf{0}} & \text { y } & \boldsymbol{\beta}_{\mathbf{1}} & \text { son } & \text { los }\end{array}$ coeficientes de las variables independientes, $\boldsymbol{u}$ es el desempleo medido por la tasa abierta de desempleo, $\boldsymbol{p}_{\boldsymbol{t}-\mathbf{1}}$ representa las expectativas adaptativas y $\boldsymbol{e}_{\boldsymbol{i}}$ es el error estocástico.

Ec. (2)

$$
\begin{gathered}
\operatorname{Ln}(\Delta p)=c-\beta_{0} u+\beta_{1} \Delta p_{t-1} \\
+e_{i}
\end{gathered}
$$

Donde $\Delta \boldsymbol{p}$ son los precios de la economía medidos por la tasa de variación interanual del índice de precios al consumidor, $\boldsymbol{C}$ es una constante, $\begin{array}{llllll}\boldsymbol{\beta}_{\mathbf{0}} & \text { y } & \boldsymbol{\beta}_{\mathbf{1}} & \text { son } & \text { los }\end{array}$ coeficientes de las variables independientes, $\boldsymbol{u}$ es el desempleo medido por la tasa abierta de desempleo, $\Delta \boldsymbol{p}_{\boldsymbol{t}-\mathbf{1}}$ representa las expectativas adaptativas y $\boldsymbol{e}_{\boldsymbol{i}}$ es el error estocástico.

Tabla No. 1 Estimaciones de la

\begin{tabular}{|c|c|c|c|c|c|c|}
\hline Ecuación & $c$ & $\beta_{0}$ & $\beta_{1}$ & $R^{2}$ & $D W$ & \\
\hline \multirow{2}{*}{ Ec. (1) } & 12.68982 & -130.3235 & 1.024106 & \multirow{2}{*}{0.998544} & 2.146015 & \\
\cline { 2 - 4 } & $(0.0000)$ & $(0.0408)$ & $(0.0000)$ & & \\
\hline \multirow{2}{*}{ Ec. (2) } & 2.336971 & -15.17403 & 4.515249 & \multirow{2}{*}{0.516537} & 1.867114 & \\
\cline { 2 - 5 } & $(0.0000)$ & $(0.0676)$ & $(0.0004)$ & & & \\
\hline
\end{tabular}

Curva de Phillips Aumentada

Nota: los valores con paréntesis son las probabilidades de cada coeficiente.

Fuente: Elaboración propia con datos de

INE y BCH

La Tabla No. 1 presenta los resultados del modelo lineal Ec. (1) y del modelo semi-logarítmico Ec. (2); el modelo lineal o Ec. (1) caracterizado por tener como variable dependiente el índice de precios al consumidor obtuvo un coeficiente de determinación $\left(\mathrm{R}^{2}\right)$ de 0.99 lo cual indica que las variables independientes (tasa desempleo e índice de precios al consumidor rezagado) explican muy bien el comportamiento de la variable dependiente, los test $\mathrm{t}$ de significancia individual también fueron favorables, demostrando la relación negativa entre preciosdesempleo, y la relación positiva de 
UN ACERCAMIENTO EMPÍRICO A LA CURVA DE PHILLIPS: HONDURAS

Revista Economía y Administración, Vol. 9, No.1, 2018

los precios respecto a los precios del pasado; por otra parte, el modelo semi-logarítmico o Ec. (2) cuya variable dependiente es la tasa de variación interanual del índice de precios al consumidor mostró un coeficiente de determinación de 0.52 valor que es consistente con las investigaciones realizadas en otros países y también indica una leve explicación por parte de las variables independientes, cabe destacar que la relación respecto al desempleo fue significativa y el coeficiente es de signo negativo lo que sustenta la hipótesis de la existencia de una relación negativa entre inflación y desempleo; es preciso mencionar que para ambos modelos se desarrolló un análisis de largo plazo y de corto plazo (Modelo de Corrección de Errores $[\mathrm{MCE}])$ realizándose pruebas de cointegración para determinar la existencia (o no) de relaciones espurias entre las variables, posteriormente se realizaron los test de normalidad, raíz unitaria, autocorrelación, heterocedastidad y variables omitidas (los resultados se pueden consultar en los anexos) obteniendo resultados consistentes y favorables en todos los test; el cálculo de la tasa natural de desempleo se obtuvo del cociente de $\frac{c}{\beta_{0}}$, esto bajo el supuesto que a largo plazo la inflación esperada es igual a la inflación observada $\left(\boldsymbol{\pi}^{\mathbf{e}}=\boldsymbol{\pi}_{\mathbf{t}}\right)$, obteniendo como resultado para el modelo lineal una TND de $9.73 \%$, mientras que para el modelo semilogarítmico se obtiene una TND del $\mathbf{1 5 . 4 \%}$, ambas TND no coinciden con los datos del desempleo del periodo de análisis, siendo necesaria la revisión de otras metodologías para cuantificar la TND, una de ellas es la aplicación del filtro HodrickPrescott.

Figura No. 3 Tasa de Desempleo y Filtro Hodrick-Prescott

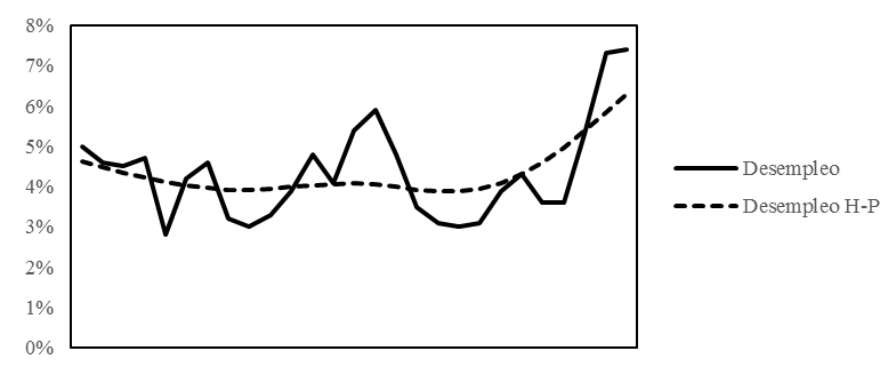

Fuente: Elaboración propia con datos del INE. 


\section{UN ACERCAMIENTO EMPÍRICO A LA CURVA DE PHILLIPS: HONDURAS}

Revista Economía y Administración, Vol. 9, No.1, 2018

Hodrick y Prescott (1997) elaboraron una metodología cuya utilidad yace en sustraer de una serie de tiempo el componente tendencial; mediante ella se determinó la tasa natural de desempleo (Figura No. 3), donde se puede apreciar que en el año de 1994 la tasa natural de desempleo fue mayor a la tasa de desempleo observada $\left(\boldsymbol{u}^{*}>\right.$ $\boldsymbol{u})$ simultáneamente hubo desequilibrios en el mercado cambiario, y esto generó incrementos en los precios de las materias primas y alimentos, por tanto, el nivel de precios aumentó; entonces, el nivel de desempleo estuvo por debajo del desempleo de tendencia, situación similar a la experimentada en 1998 cuando los desastres climatológicos distorsionaron la actividad económica por el incremento de precios; de manera distinta en 2016.

El nivel de precios tuvo un crecimiento inferior a la meta de inflación, sin embargo, el desempleo se situó por encima de su tendencia de largo plazo $\left(\boldsymbol{u}^{*}<\boldsymbol{u}\right)$, esto puede ser ocasionado por una multiplicidad de variables exógenas o estructurales inherentes a la economía hondureña; de acuerdo con las afirmaciones anteriormente expuestas se puede mejorar la situación del mercado laboral abogando por políticas que estimulen el nivel de empleo sin alterar el nivel de precios.

Figuras No. 4 y No. 5 Estimaciones:

Ec. (1) y Ec. (2

Fuente: Elaboración propia con datos del
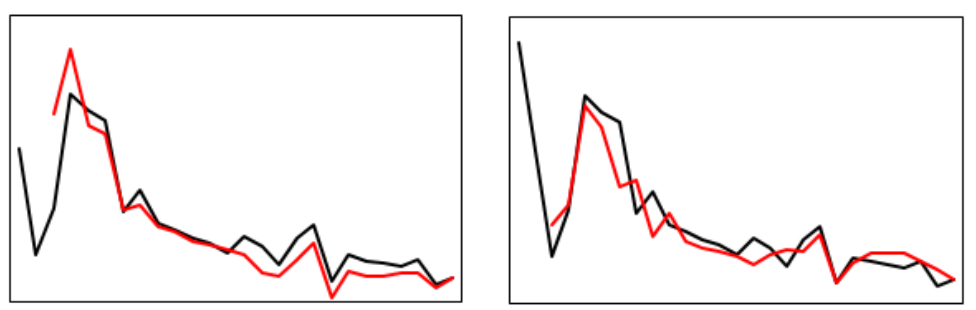

INE y BCH

Ambas Figuras (No. 4 y No. 5) son producto de una comparación de la inflación efectiva (color negro) y la inflación estimada (color rojo) elaborada por los modelos de curva de Phillips con expectativas; se puede observar en cierta medida que la inflación estimada tiene un comportamiento semejante a la inflación efectiva, sin embargo, al ser la inflación un indicador volátil o sensible a choques externos requiere de una estimación más incluyente en términos de variables, puesto que la inflación depende no solo del desempleo y de las expectativas. 


\section{UN ACERCAMIENTO EMPÍRICO A LA CURVA DE PHILLIPS: HONDURAS}

Revista Economía y Administración, Vol. 9, No.1, 2018

Tabla No. 2 Prueba de Causalidad de

Granger

\begin{tabular}{|c|c|c|c|}
\hline Ecuación Rezago & 1 Rezage & 2 $\underline{\text { Rezagos }}$ & 3 $\underline{\underline{\text { Rezagos }}}$ \\
\hline Inflación $\rightarrow$ Desempleo & 0.4196 & 0.8086 & 0.5846 \\
\hline Desempleo $\rightarrow$ Inflación & 0.5631 & 0.8086 & 0.2906 \\
\hline$I P C \rightarrow$ Desempleo & 0.0971 & 0.2771 & 0.4361 \\
\hline Desempleo $\rightarrow I P C$ & 0.0407 & 0.2172 & 0.1994 \\
\hline
\end{tabular}

Nota: Los números en la Tabla 2 son las probabilidades de cada test. Fuente: Elaboración propia con datos del INE y BCH.

Para evitar afirmaciones erróneas se elaboró el test de causalidad de Granger (1969) para determinar si existe una relación causal entre inflación y desempleo, este test consiste en elaborar un pronóstico para una variable "a" en función de los rezagos de la misma variable y de una variable "b"; los resultados de dicho test demostraron (salvo para los casos de Desempleo $\rightarrow$ IPC e IPC $\rightarrow$ Desempleo con un rezago, ya que con un alfa de $10 \%$ muestran una causalidad bidireccional) que hay independencia causal en el sentido de Granger entre inflación y desempleo, sin embargo, ambas variables presentan una correlación negativa, esto comprobado a través de los modelos econométricos de curva de Phillips con expectativas realizados. (Tabla No. 2)

\section{CONCLUSIONES}

En base al ejercicio econométrico realizado y analizando el comportamiento de las variables en el tiempo se puede afirmar que en Honduras se da lugar a una relación negativa entre inflación y desempleo, esto demostrado por el signo de los coeficientes y por el test de significancia individual que tanto en el modelo lineal como en el modelo semi-logarítmico comprobaron la tesis de la existencia de una relación inversa.

La estimación presentó resultados que hasta cierto punto apoyan o refuerzan el argumento de la curva de Phillips (en el sentido de una relación negativa entre ambas variables), sin embargo, y como se expuso anteriormente la inflación es una variable volátil que depende tanto de variables endógenas como exógenas, si bien es cierto la curva de Phillips es un instrumento de análisis importante para los hacedores de política, es pertinente hacer estudios más profundos sobre la misma y los diferentes planteamientos que se han desarrollado entorno a ella.

La aproximación empírica realizada de la tasa natural de desempleo en 


\section{UN ACERCAMIENTO EMPÍRICO A LA CURVA DE PHILLIPS: HONDURAS}

Revista Economía y Administración, Vol. 9, No.1, 2018

Honduras obtuvo resultados mixtos, por una parte, utilizando la ecuación lineal y semi-logarítmica los resultados estuvieron por encima del desempleo observado en el periodo de análisis y poco cercanos a cualquier valor observado en los últimos 25 años, por otra parte, la metodología empleada utilizando el filtro Hodrick-Prescott arrojó valores que oscilan entre el $4 \%$ y $5 \%$ con la salvedad de los últimos dos años, donde se manifestaron valores superiores a dicho intervalo; en conclusión los resultados obtenidos por el filtro Hodrick-Prescott son más próximos a la tasa de desempleo observada y por ende es una aproximación empírica más cercana al concepto de TND.

Alejándose del análisis positivo se puede concluir que la curva de Phillips con expectativas no es el mejor instrumento para la dirección y manejo de política económica dada la inconsistencia estadística presentada en algunas etapas de la elaboración de los modelos econométricos y de las pruebas de causalidad, si bien es cierto existe una relación negativa entre inflación y desempleo (sustentada en la teoría y la práctica), no quiere que decir que una variable determine otra. Finalmente, dado el nivel de desempleo y el nivel de precios en los últimos años se puede concluir que el uso de políticas encaminadas a reducir el desempleo no provocará impactos alarmantes en el nivel de precios.

\section{REFERENCIAS}

CLARIDA, R., GALÍ, J. and GERTLER, M. 1999. The science of monetary policy: a new keynesian perspective. Journal of Economic Literature, 37(2), 1661-1707.

FRIEDMAN, M. 1968. The role of monetary policy. The American Economic Review, 58(1), 1-17.

FRIEDMAN, M. 1977. Nobel lecture: inflation and unemployment. Journal of Political Economy, 85(3), 451-472.

GALÍ, J. and GERTLER, M. 1999. Inflation dynamics: a structural econometric analysis. Journal of Monetary Economics, 44(2), 195222.

GRANGER， C.W.J. 1969. Investigating causal relations by econometric models and cross- 
UN ACERCAMIENTO EMPÍRICO A LA CURVA DE PHILLIPS: HONDURAS

Revista Economía y Administración, Vol. 9, No.1, 2018

spectral methods. Econometrica, 37(3), 424-438.

HENAO, M.L. and ROJAS, N. 1998.

La tasa natural de desempleo en Colombia. Coyuntura Económica, 29(3), 79-93.

HODRICK, R.J. and PRESCOTT, E.C. 1997. Postwar U.S. business cycles: an empirical investigation. Journal of Money, Credit and Banking, 29(1), 1-16.

MODIGLIANI, F. and PAPADEMOS, L. 1975. Targets for monetary policy in the coming year. Brookings Papers on Economic Activity, 6(1), 141-165.

PHELPS, E.S. 1968a. Money-wage dynamics and labor-market equilibrium. Journal of Political Economy, 76(4), 678-711.

PHELPS, E.S. 1968b. Phillips curves, expectations of inflation and optimal unemployment over time: reply. Economica, 35(139), 288-296.

PHILLIPS, A.W. 1958. The relation between unemployment and the rate of change of money wage rates in the
United Kingdom, 18611957. Economica, 25(100), 283-299.

ROBERTS, J. 1995. New keynesian economics and the Phillips curve. Journal of Money, Credit and Banking 27(4), 975-984.

SAMUELSON, P.A. and SOLOW, R.M. 1960. Analytical aspects of anti-inflation policy. The American Economic Review, 50(2), 177-194.

STIGLITZ, J. 1997. Reflections on the natural rate hypothesis. The Journal of Economic Perspectives, 11(1), 3-10.

SUÁREZ, V. B. P. and EDUARDO, N. C. R. 2011. Curva de phillips y la tasa natural de desempleo. Pensamiento Crítico, 16, 79-93.

TOBIN, J. 1980. Stabilization policy ten years after. Brookings Papers on Economic Activity, 11(1), 19-71.

TOBIN, J. 1997. Supply constraints on employment and output: NAIRU versus natural rate. Cowles Foundation-Yale University, (1150). 1-24. 
UN ACERCAMIENTO EMPÍRICO A LA CURVA DE PHILLIPS: HONDURAS

Revista Economía y Administración, Vol. 9, No.1, 2018 